\title{
Numerical simulation of electrohydraulic processes
}

\author{
Viatcheslav S. Mamutov ${ }^{1, *}$ and Alexander V. Mamutov ${ }^{2}$ \\ ${ }^{1}$ Peter the Great St. Petersburg Polytechnic University, Polytechnicheskaya 29, St. Petersburg, 195251, \\ Russian Federation \\ ${ }^{2}$ Valiant Int., 2469 Executive Hills Dr, Auburn Hills, MI 48326, USA
}

\begin{abstract}
Using the finite element software complex LS-DYNA 971, the technique of simulating processes of Electro-Hydraulic forming is developed. The law of energy deposition is obtained from semi-analytical calculation. A simulation of complex EHF process, which includes discharge chamber with electrodes, plasma channel, water, deformable blank that is formed into a rigid die, is performed using the developed technique. The simulation is validated by experimental results. The developed technique significantly extends practical usage of numerical simulation of electrohydraulic forming and other electrohydraulic-based technologies.
\end{abstract}

\section{Introduction}

The electrohydraulic effect, a combined effect of shock waves and streams generated during a high-voltage electric discharge in liquid, finds wide use in the various processes of ElectroHydraulic Forming (EHF) of automotive parts and in other areas of manufacturing [1-6]. High-voltage electric discharge in liquid is also used in other technologies: for the purpose of materials breaking, granulating and shredding, notably for medical purposes in treatment of the kidney stone disease; for intensification of chemical and physical processes; in mining of minerals and in some other applications [7]. Relatively short pressure front close to the channel $(0.1-10 \mu \mathrm{s})$, high pressure amplitude (up to few GPa) with relatively long total duration of the process (up to few $\mathrm{ms}$ ), and also the accompanying non-linear effects in liquid and in the interacting objects create particular requirements to the stability of numerical algorithms used in simulation of such processes.

Some assumptions were made for simulating EHF process: regarding discharge current at variable electric resistance of the discharge channel, initiating and expanding the plasma channel, propagating of the pressure wave in the liquid, loading and deforming of the workpiece, similar to that made in $[8,9]$.

Currently, one of the most efficient Finite Element (FE) software complexes for computer simulation of a high-voltage discharge in liquids is LS-DYNA [10]. This software can simulate interacting of several fluids and deformable solids objects through using the Arbitrary Lagrangian Eulerian (ALE) algorithm which, in combination with Multi-Material

*Corresponding author: mamutov_vs@spbstu.ru 
technique and Fluid-Structure Interaction (FSI) technique, eliminates the problem of mesh distortion and allows solving the problem of moving liquids and gases inside of a closed complex shape volume [11]. In the context of EHF, a simulation of a discharge channel in liquid for the case of cylindrical symmetry was done in work [12], and for the case of a chamber with coaxial closely placed electrodes - in works [13-16]. In these works, electric power laws were used to provide input in LS-DYNA for energy deposit laws into the discharge channel. The functions were experimentally obtained. To improve practical usage of EHF simulation, it is beneficial to do complex simulation using calculated electric power law.

The goal of this work was creating a complex computer model of EHF process using the software complex LS-DYNA 971 together with calculated function of electric power law as energy deposition into the discharge channel, and also to estimate the influence of numerical parameters on results of simulation.

\section{Simulation of electrical circuit and plasma}

The scheme of electro-hydraulic-impulse loading is presented in fig. 1, $a$. The capacitor battery $C$ of electro-impulse unit (pulse generator 1 ) is charging up to some high voltage from step-up transformer $T$ and high-voltage rectifier $R_{c}, R$. When battery discharges through controlled discharger $D$ the electrical breakdown of interelectrode gap of electrode system 4 in liquid 3 in discharge chamber 2 occurs. Next explosive expansion of plasma gas cavity due to adiabatic heat by impulse current occurs, that leads to compression in a liquid. Impulse pressure provides the required action, for example forming the sheet metal blank 5 into the die 6.

a)

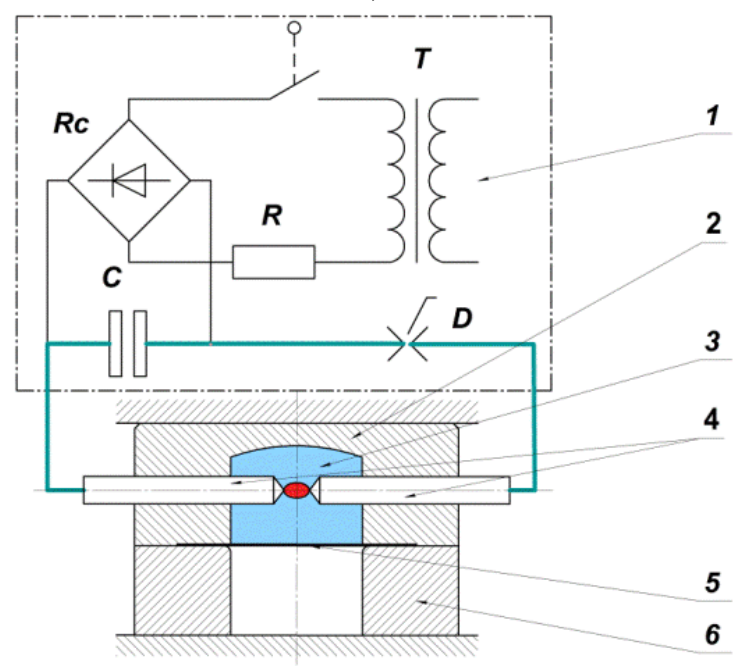

3

4 6 b)

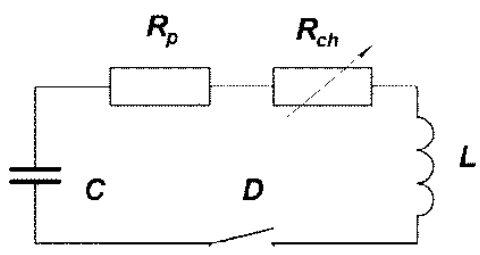

Fig. 1. Scheme of electro-hydraulic forming: $a$-process scheme: 1 - pulse generator, 2 - discharge chamber, 3 - pressure transmitting liquid, 4 - electrodes, 5 - blank, 6 -die; $b$ - equivalent scheme of discharge circuit

From the breakdown moment via the interelectrode gap, which is a low-temperature highpressure plasma, the discharge of capacitor battery started (fig. $1, b$ ). Here $R_{p}$ - parasitic resistance of discharge circuit, $R_{c h}$ - resistance of discharge channel, $L$ - inductance of discharge channel. Computational problem is defined as a set of two mutually bounded 
problems: the first is a process in electrical circuit, discharge of capacitor through plasma gap with varying resistance; the second is a hydrodynamic problem, expansion of a gas-vapor cavity due to release of electrical energy and rising of pressure waves and a high-speed plasticity blank deformation under the action of an impulse pressure.

The system of the equations for the plasma channel was made mainly on the basis of E.V. Krivickiy theories $[17,18]$.

Differential equation for current $i(t)$ in a circuit (fig. 2) with varying resistance of discharge channel $R_{c h}$, with combined own resistance of electro-impulse unit and contacts $R_{p}$, and inductance of discharge circuit $L$ is given by

$$
L \frac{d^{2} i}{d t^{2}}+\frac{d}{d t}\left\{\left[R_{c h}(i, t)+R_{p}\right] i\right\} \cdot+\frac{1}{C} i=0,
$$

under the initial conditions

$$
i(0)=0, \frac{d i}{d t}=-\frac{U_{0}}{L},
$$

where $U_{0}$ - voltage of charge capacitor battery $C$.

Resistance of discharge channel $R_{c h}$ depends on conductivity of plasma

$$
R_{c h}=\frac{1}{\sigma} \frac{l_{c h}}{\pi \cdot r_{c h}^{2}},
$$

where $l_{c h}, r_{c h}$ - length and radius of plasma channel, conductivity of plasma $\sigma$ can be defined by Spitzer formula

$$
\sigma=0.00152 \frac{T^{3 / 2}}{\ln \Lambda},
$$

where $T$-temperature of plasma, and when $1 \cdot 10^{4} \leq T \leq 3 \cdot 10^{4}(K)$. It is a characteristic of the $E H F$ processes, because temperature of plasma significantly depends on parameters of discharge circuit and on the discharge conditions. Therefore coulomb logarithm is within the limits of: $1.1 \leq \ln \Lambda \leq 1.9$, and it is possible to accept a mean $\ln \Lambda \cong 1.5$.

When $l_{c h}=10 \mathrm{~mm}$ (initiating wire is used), capacitance of battery $C=600 \mu \mathrm{F}$, inductance of the circuit $L=1.75 \mu \mathrm{H}$, parasitic electric resistance $R_{p}=0.002 \Omega$, the law of dimensionless electric power vs. dimensionless time looks like follows (Fig. 2).

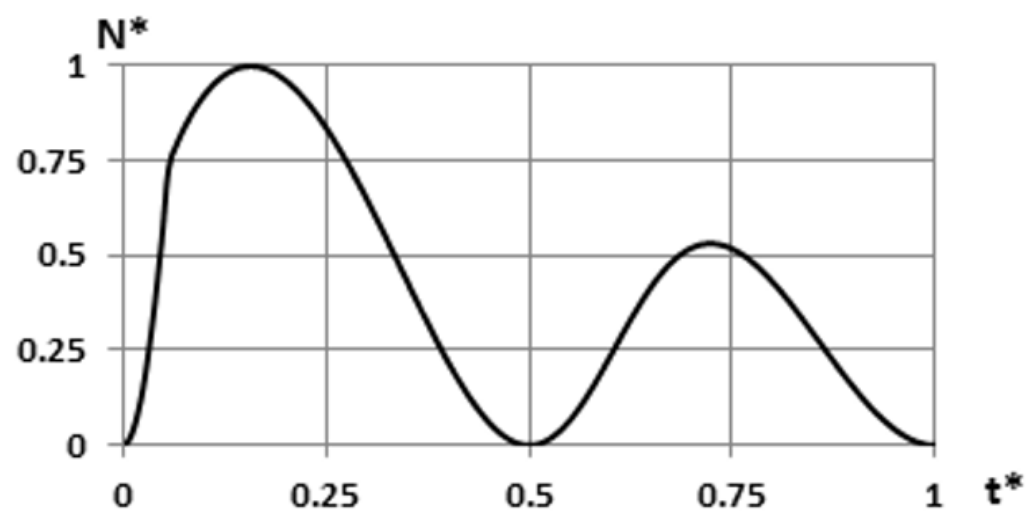

Fig. 2. Energy deposit law. $N^{*}=N / N_{\max }, t^{*}=t / T_{0}$ $N_{\max }=4.9 \mathrm{MW}, T_{0}=101.8 \mu \mathrm{s}$

This law is very close to the experimental one obtained in [12] at the same parameters of the process. 


\section{Specifics of simulating discharge}

Some specifics related to simulating a discharge in a discharge chamber of real shape are given below. Despite all the measures, the attempts to build a numerical model using Lagrangian approach were unsuccessful. To improve stability of the model the $A L E$ Smoothing technique was used that can stabilize the mesh inside of a particular Lagrangian body. Even with this measure the mesh is distorting, especially in the area of contact of two bodies due to the lack of shear rigidity of fluid elements. Because of distortion and subsequent numerical instability, the achieved simulation time using this approach usually did not exceed 100-200 $\mu$ s. There are also difficulties in defining contact between Lagrangian plasma body and other objects using both the Penalty Based and Constraint Based contact algorithms.

Using Multi-Material ALE approach allows simulating complex shape chamber and electrodes geometries with long simulation time. The example of such simulation is shown in Fig. 3.

a)

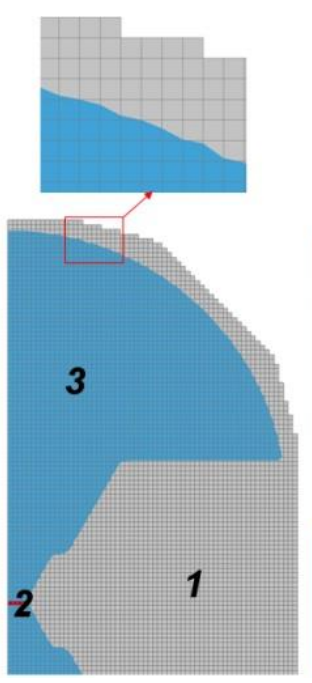

b)

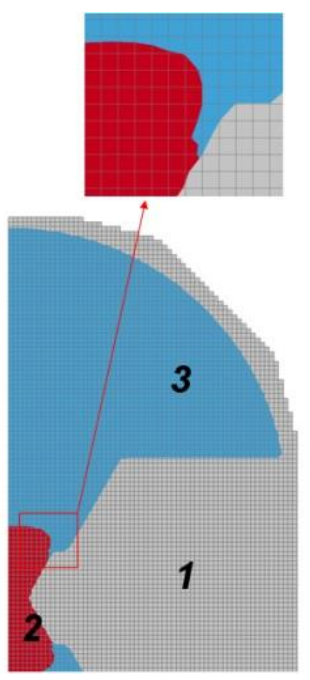

Fig. 3. Example of simulation of the discharge channel in 3-dimensional discharge chamber: (the cross-section along the electrodes is shown) at the initial moment $(a)$ and during the expanding channel $(b)$

As it is shown, the material of movable media (liquid 3 and plasma 2) is moving through the stationary regular mesh (vacuum mesh 1 ). There can be more than one physical material inside of one ALE mesh cell. Such an approach allows avoiding time consuming adaptation of mesh to a particular geometry of rigid bodies and eliminate the problem of mesh distortion during simulation. Also the contact between fluids does not require special definition and works automatically as a part of ALE formulation. The contact between fluids and solids (FSI - Fluid-Structure Interaction) in LS-DYNA is still based on the penalty factor algorithm, but is significantly improved in comparison with that in Lagrangian formulation and stable in a long range of density and pressure of the moving media. 


\section{Computer model and results of simulation}

The materials of the electrodes and the chamber walls were defined as rigid. The $M A T \_R I G I D$ card was used.

Equation of state for water is given by the form of Taite adiabat [20]

$$
p=p_{o}+B\left[\left(\rho / \rho_{o}\right)^{n}-1\right],
$$

where $p_{\mathrm{o}}$ - barometric pressure, parameters of adiabat for tap water are: $\rho$ - liquid density, $\rho_{\mathrm{o}}$ $=1000 \mathrm{~kg} / \mathrm{m}^{3}, B=304.7 \mathrm{MPa}, n=7.15$. To simulate water in LS-DYNA, the liquid was assumed an ideal compressible fluid. The MAT_ELASTIC_FLUID card was used which defines pressure in the liquid as

$$
p=-K \cdot \ln \left(\rho_{0} / \rho\right) \text {. }
$$

The bulk modulus $K$ is defined from approximation of Tait adiabat for water in the pressure range $p \in[0.1-100] \mathrm{MPa}$, which gives approximate value of $K \cong 2.35 \mathrm{GPa}$. The cavitation threshold is defined as $0.1 \mathrm{MPa}$.

For the deformable part, the MAT_POWER_LAW_PLASTICITY card with following parameters of hardening curve was used: $B=1357 \mathrm{MPa}, m=0.29$. The elastic properties of the material were defined as: $E=202 \mathrm{GPa}$ - elastic modulus, $v=0.31$ - Poisson's ratio, density $\rho=7800 \mathrm{~kg} / \mathrm{m}^{3}$. Coulomb coefficient of friction was $\mu=0.2$ for static friction and $\mu=0.15$ for sliding friction. The binder was gap controlled with constant gap $0.21 \mathrm{~mm}$.

Energy equation in plasma gas cavity generally is given by [19]

$$
N_{c h}(t)=\frac{1}{\gamma-1}\left(V_{c h} \frac{d p_{c h}}{d t}+\gamma p_{c h} \frac{d V_{c h}}{d t}\right)
$$

where $N_{c h}(t)$ - electrical power in the channel

$$
N_{c h}=i(t)^{2} \cdot R_{c h}(t)
$$

$\gamma=1.26$ - ratio for plasma obtained from a tap water, $V_{c h}$ and $p_{c h}$ - channel volume and channel pressure. According to research done in [19], the power losses from radiation and the power spent on expulsion of water on a wall of the channel can be neglected when keeping practical accuracy in typical conditions of EHF.

To simulate the plasma channel the MAT_NULL card was used for the plasma material. It defines the initial plasma density $\rho_{p 0}$ as well as an equation of state using the EOS_LINEAR_POLYNOMIAL_WITH_ENERGY_LEAK card that defines the equation of energy balance that corresponds to (7) and the energy deposition law (8) defined by the graph in Fig. 2. Simulation was performed using ALE solver. To reduce simulation time and because of symmetry of the workpiece and the chamber, only one quarter of the simulated area was meshed.

In Fig. 4 the discharge channel shape, shape of the fluid fractions and the blank at $120 \mu \mathrm{s}$ $(a)$, and also the distribution of pressure in the chamber are shown at $80 \mu \mathrm{s}(b)$. 
a)

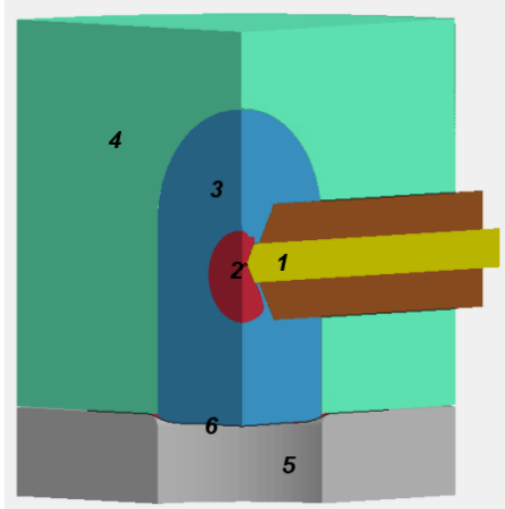

b)

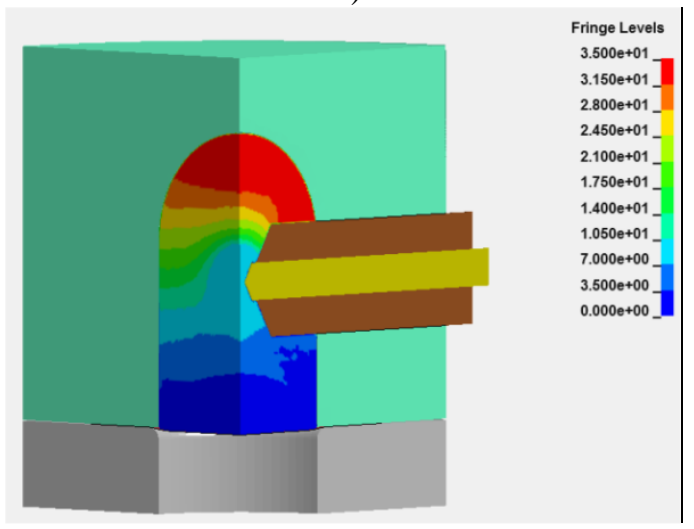

Fig. 4. Deformation of the fluid parts and the blank at $120 \mu \mathrm{s}(a)$ :

1 - electrode, 2 - plasma bubble, 3 - water, 4 - discharge chamber, 5 - die, 6 - deforming blank and distribution of the pulse pressure at $80 \mu \mathrm{s}(b)$

The shape of the blank after finishing deformation is shown in Fig. 5, $a$. In Fig. 5, $b$ plastic deformation vs. time is shown for the marked elements.

a)

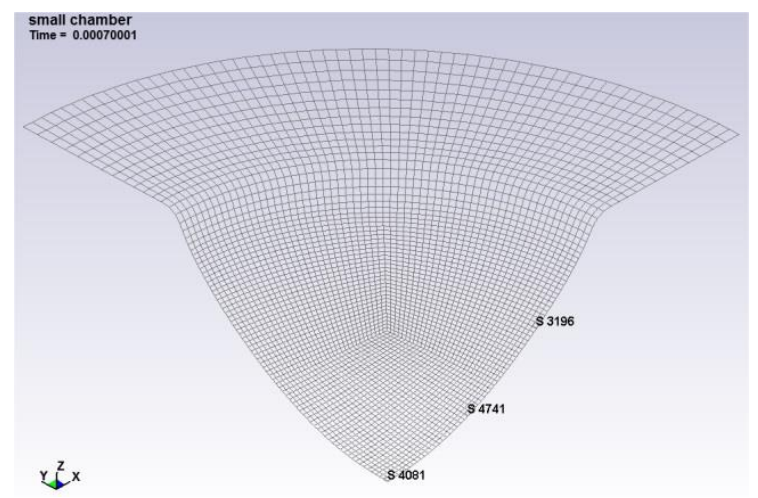

b)

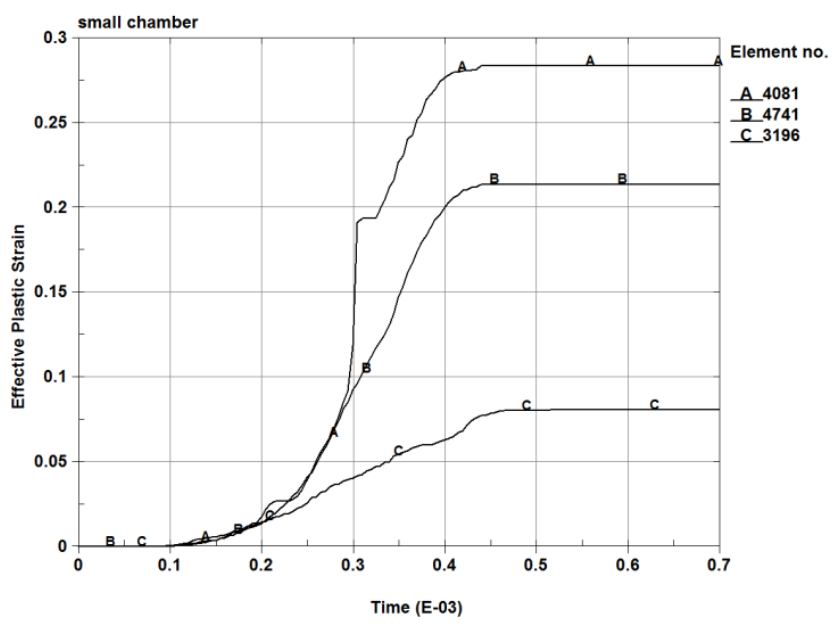

Fig. 5. Deformation of the blank: $a$ - the final shape of the blank; $b$-effective plastic strain vs. time for the marked elements. 
The results of simulation presented in Fig. 4 are used for comparison with the experimental data on maximum deformation of the blank and on the plastic strain in the center of the blank. For the given variant of the process parameters, the difference of experimental data and simulation on maximum move of the blank's center is about $20 \%$ and on effective plastic strain at the center is about $25 \%$.

\section{Conclusion}

Using the finite element software complex LS-DYNA 971, the technique of simulating processes of Electro-Hydraulic forming is developed. The law of energy deposition is obtained from semi-analytical calculation based on E.V. Krivickiy theories. The obtained law is very close to the experimental one obtained in previous researches at the same parameters of the process. A simulation of complex EHF process, which includes discharge chamber with electrodes, plasma channel, water, deformable blank that is formed into a rigid die, is performed using the developed technique. The simulation is validated by experimental results. The developed approach doesn't require experimenting for obtaining energy deposit law. Therefore, it significantly extends practical usage of numerical simulation of electrohydraulic forming and other electrohydraulic-based technologies.

\section{References}

1. G.A. Guly (ed), Equipment and technological processes using electro-hydraulic effect (Mechanical Engineering, Moscow, 1977)

2. V.N. Chachin et al., Electrohydroimpulse processing of materials in mechanical engineering (Science and technology, Minsk, 1987)

3. M.E. Taranenko, Electro-hydraulic forming: theory, equipment, and processes (Publishing House of HAI, 2011)

4. G.N. Zdor, L.A. Isaevich, I.V. Kachanov, Technology high-speed deformation of metals (Publishing House of BNTU, Minsk, 2010)

5. A. Rohatgi. E.V. Stephens, R.W. Davies, M.T. Smith, A. Soulami, S. Ahzi, J. Mater. Process. Tech., 212 1070-1079 (2012)

6. S. Golovashchenko, V. Mamutov, Sixth Global Innovations Symposium: Trends in Materials and Manufacturing Technologies for Transportation Industries. Proc. of TMS, 65-70 (San Francisco, 2005)

7. G.A. Guliy, P.P. Maliushevskiy, High-voltage pulsed discharge in pulsed power systems (Naukova Dumka, Kiev, 1977)

8. V.A. Pozdeev, Applied hydrodynamics of the electrical discharge of the liquid (Naukova Dumka, Kiev, 1980)

9. G.N. Gavrilov et al., Discharge pulse technology (Naukova Dumka, Kiev, 1979)

10. J.O. Hallquist, LS-DYNA theoretical manual (Livermore Software Technology Corporation, Livermore, CA, 2006)

11. M. Souli, D.J. Benson (ed), Arbitrary Lagrangian and Fluid-Structure Interaction. Numerical Simulation (ISTE, Great Britain and the United States, 2010)

12. S.F. Golovashchenko, A.V. Mamutov, V.S. Mamutov, St. Petersburg State Polytechnical University Journal, 1 (147) 116-121 (2012)

13. A.V. Mamutov, V.S. Mamutov, St. Petersburg State Polytechnical University Journal, 1 (190) 101-107 (2014) 
14. V. Mamutov, A. Mamutov, S. Golovaschenko, 13th International LS-DYNA ${ }^{\circledR}$ Users Conference, Metal forming, 9 (Dearborn, USA, June 8-10, 2014)

15. A.V. Mamutov, S.F. Golovashchenko, V.S. Mamutov, J.J.F. Bonnen, J. Mater. Process. Tech., 219, 84-100 (2015)

16. V.S. Mamutov, G.N. Zdor, Int. Rev. Mech. Eng., 11(5) 332-336. (2017)

17. E.V. Krivitskii, V.V. Shamko, A.I. Vovchenko, Pulse-discharge technology (Naukova Dumka, Kiev, 1978)

18. E.V. Krivickiy, V.V. Shamko, Transients in high-voltage discharge in a fluid (Naukova Dumka, Kiev, 1979)

19. K.A. Naugolnyh, N.A. Roy, Electrical discharges in water.(Science, Moscow, 1971)

20. R. Cole, Underwater explosions (Foreign Literature Publishing House, Moscow, 1950) 